\title{
REFRACTION OF ACOUSTIC DUCT WAVEGUIDE MODES BY EXHAUST JETS*
}

\author{
BY \\ R. MANI \\ General Electric Research and Development Center, Schenectady
}

\begin{abstract}
The refraction of acoustic duct waveguide modes emitted from the open end of a semi-infinite rectangular duct by a jet-like exhaust flow is studied theoretically. The problem is formulated as a Wiener-Hopf problem and is ultimately solved by an approximate method due to Carrier and Koiter. Continuity of transverse acoustic particle displacement and of acoustic pressure is assumed at the jet/still-air interface. The solution exhibits several features of the acoustics of moving media such as a source convection effect, zones of relative silence, simple refraction, etc. Plots of far-field directivity patterns are presented for several cases and show refraction effects to be important even at modest exhaust Mach numbers of order 0.3 . Only subsonic exhaust Mach numbers are considered. In view of the problem's technological interest, the solution for the far-field directivity is written out in full detail in the appendices. In the low-frequency limit when only one duct waveguide mode (the plane wave mode) propagates, we also examine the reflection coefficient. It is found that this reflection coefficient, in general, considerably exceeds either the no-flow value or the value for the case with uniform flow both inside and outside the waveguide (i.e., in the whole space). Apparently the acoustic medium mismatch enhances the geometric mismatch in the jet flow case, thus producing a higher reflection coefficient.

1. Formulation of problem. A slug-like jet flow of uniform subsonic Mach number $M$ issues from a pair of parallel plates spaced a distance $2 b$ apart (Fig. 1). The regions $y>b$ and $y<-b$ are occupied by still fluid at the same temperature and static pressure as the jet. An acoustic duct waveguide mode with a transverse pressure distribution of type $\cos (N \pi(y-b) / 2 b) \exp (-j \omega t)$, where $j=\sqrt{ }-1$, is incident from $x=-\infty$ within the pair of parallel plates. The problem is to determine the far-field acoustic radiation pattern produced by the emission of such a mode.

The case when there is no flow has been fully solved by Noble [5], amongst other authors. The problem with $N=0$ and with a circular tube waveguide instead of a pair of parallel plates was considered by Carrier [1]. However, Carrier employed continuity of transverse acoustic velocity as a boundary condition on $y= \pm b$ and $x>0$; this is now believed to be incorrect. When one has mean flow velocity discontinuities it is presently accepted (Ribner [6], Gottlieb [3]) that the transverse acoustic particle displacement should be continuous. Also, Carrier [1] was more interested in the reflection coefficient in this problem and did not obtain any explicit solution.
\end{abstract}

* Received October 20, 1971. 
In mathematical terms, with the usual assumptions of linear, inviscid acoustics and assuming for all quantitics a time dependence of type exp $(-j \omega t)$, and with reference to Fig. 1 , we wish to find a velocity potential $\phi$ such that

a) in regions I, III, $\phi$ satisfies:

$$
\nabla^{2} \phi+k^{2} \phi=0
$$

where $k=\omega / c$.

b) in region II it satisfies:

$$
\left(1-M^{2}\right)\left(\partial^{2} \phi / \partial x^{2}\right)+\left(\partial^{2} \phi / \partial y^{2}\right)+2 j M k(\partial \phi / \partial x)+k^{2} \phi=0 .
$$

c) at $y= \pm b, \partial \phi / \partial y=0$ for $x<0$.

d) (continuity of acoustic pressure) for $x>0$,

$$
\begin{aligned}
& \text { at } y=b: \quad \phi]_{y=b+}=\left[\phi+j \frac{M}{k} \frac{\partial \phi}{\partial x}\right]_{y=b-} \\
& \text { at } \left.y=-b:\left[\phi+j \frac{M}{k} \frac{\partial \phi}{\partial x}\right]_{y=-b+}=\phi\right]_{y=-b-} .
\end{aligned}
$$

e) (continuity of transverse acoustic particle displacement) at $y= \pm b$ : for $x>0, \eta$, the transverse acoustic particle displacement, is continuous. The relation between $\eta$ and $\phi$ in regions I, III is:

$$
\eta=(j / \omega)(\partial \phi / \partial y)
$$

and in region II is:

$$
[\eta+j(M / k)(\partial \eta / \partial x)]=(j / \omega)(\partial \phi / \partial y) .
$$

2. Fourier integral representation and application of Wiener-Hopf technique. In what follows the notation has been arranged to conform closely to that used by Noble [5]. Clearly, from the symmetry of the problem it is sufficient to consider regions I, II alone.

Adopt for $\phi$ the integral representation in region I of type:

$$
\phi=\frac{-1}{(2 \pi)^{1 / 2}} \int_{-\infty}^{\infty} \frac{A(\alpha) \exp (\gamma(b-y) \exp (-j \alpha x) d \alpha}{\gamma},
$$

where $\gamma=\left(\alpha^{2}-k^{2}\right)^{1 / 2} \cdot \gamma$ has branch points at $\alpha= \pm k$ and branch cuts are introduced joining $\pm k$ to $\infty$. When $\alpha$ is real and $|\alpha|<k, \gamma=-j\left(k^{2}-\alpha^{2}\right)^{1 / 2}$. Similarly, in region II adopt the representation:

$$
\phi=\frac{1}{(2 \pi)^{1 / 2}} \int_{-\infty}^{\infty} \frac{B(\alpha)\left\{\begin{array}{l}
\cosh \left(\gamma^{\prime} y\right) \\
\sinh \left(\gamma^{\prime} y\right)
\end{array}\right\} e^{-i \alpha x} d \alpha}{\gamma^{\prime}\left\{\begin{array}{l}
\sinh \left(\gamma^{\prime} b\right) \\
\cosh \left(\gamma^{\prime} b\right)
\end{array}\right\}} .
$$

$\gamma^{\prime}=\left(\alpha^{2}-(k+\alpha M)^{2}\right)^{1 / 2}$ and has branch points at $\alpha=k /(1-M)$ and $\alpha=-k /(1+M)$. Branch cuts are introduced joining the points $k /(1-M)$ and $-k /(1+M)$ to $\infty$ and when $\alpha$ is real and $-k /(1+M)<\alpha<k /(1-M), \gamma^{\prime}=-j\left[(k+\alpha M)^{2}-\alpha^{2}\right]^{1 / 2}$. The combination $\cosh \left(\gamma^{\prime} y\right) / \sinh \left(\gamma^{\prime} b\right)$ will be used in the integrand if $N$ is even and and the combination $\sinh \left(\gamma^{\prime} y\right) / \cosh \left(\gamma^{\prime} b\right)$ if $N$ is odd. 
The associated acoustic pressure $p$, transverse velocity $v$, and transverse particle displacement $\eta$ are given by:

a) in region $\mathrm{I}(y>b)$ :

$$
\begin{aligned}
& p=\frac{-j \omega \rho_{0}}{(2 \pi)^{1 / 2}} \int_{-\infty}^{\infty} \frac{A(\alpha) \exp (\gamma(b-y)-j \alpha x) d \alpha}{\gamma}, \\
& y=\frac{1}{(2 \pi)^{1 / 2}} \int_{-\infty}^{\infty} A(\alpha) \exp (\gamma(b-y)-j \alpha x) d \alpha, \\
& \eta=\frac{1}{\omega(2 \pi)^{1 / 2}} \int_{-\infty}^{\infty} A(\alpha) \exp (\gamma(b-y)-j \alpha x) d \alpha .
\end{aligned}
$$

b) in region II $(-b \leq y \leq b)$ :

$$
\begin{aligned}
& p=\frac{j \omega \rho_{0}}{(2 \pi)^{1 / 2}} \int_{-\infty}^{\infty} \frac{B(\alpha)\left(1+\frac{M \alpha}{k}\right)\left\{\begin{array}{l}
\cosh \left(\gamma^{\prime} y\right) \\
\sinh \left(\gamma^{\prime} y\right)
\end{array}\right\} e^{-j \alpha x} d \alpha}{\gamma^{\prime}\left\{\begin{array}{l}
\sinh \left(\gamma^{\prime} b\right) \\
\cosh \left(\gamma^{\prime} b\right)
\end{array}\right\}}, \\
& v=\frac{1}{(2 \pi)^{1 / 2}} \int_{-\infty}^{\infty} \frac{B(\alpha)\left\{\begin{array}{l}
\sinh \left(\gamma^{\prime} y\right) \\
\cosh \left(\gamma^{\prime} y\right)
\end{array}\right\} e^{-i \alpha x} d \alpha}{\left\{\begin{array}{l}
\sinh \left(\gamma^{\prime} b\right) \\
\cosh \left(\gamma^{\prime} b\right)
\end{array}\right\}}, \\
& \eta=\frac{j}{\omega(2 \pi)^{1 / 2}} \int_{-\infty}^{\infty} \frac{B(\alpha)\left\{\begin{array}{l}
\sinh \left(\gamma^{\prime} y\right) \\
\cosh \left(\gamma^{\prime} y\right)
\end{array}\right\} e^{-j \alpha x} d \alpha}{M \alpha}\left\{\begin{array}{l}
\sinh \left(\gamma^{\prime} b\right) \\
\cosh \left(\gamma^{\prime} b\right)
\end{array}\right\}
\end{aligned}
$$

Clearly the integral representations for $\phi$ ensure satisfaction of (1), (2).

The integrals for $\phi, p, v$ and $\eta$ could be evaluated by closing the contour in the $\alpha$ plane. For $x<0$, one would close the contour by means of a large semicircle in the upper halfplane and for $x>0$, a large semicircle in the lower halfplane would be employed.

Let the wave incident from within the duct from $x=-\infty$ be of form

$$
\phi_{\mathrm{inc}}=\exp \left(j K_{N} x\right) \cos [N \pi / 2 b(y-b)],
$$

where

$$
K_{N}=\frac{-k M+\left[k^{2}-(N \pi / 2 b)^{2}\left(1-M^{2}\right)\right]^{1 / 2}}{\left(1-M^{2}\right)}
$$

The associated pressure, transverse velocity, and transverse acoustic particle displacement fields are:

$$
\begin{aligned}
& p_{\text {inc }}=j \rho_{0} \omega\left(1-\frac{M K_{N}}{k}\right) \exp \left(j K_{N} x\right) \cos \left(\frac{N \pi}{2 b}(y-b)\right), \\
& v_{\text {ino }}=-\frac{N \pi}{2 b} \exp \left(j K_{N} x\right) \sin \left[\frac{N \pi}{2 b}(y-b)\right],
\end{aligned}
$$




$$
\eta_{\mathrm{inc}}=\frac{-j}{\omega}\left(\frac{N \pi}{2 b}\right) \frac{\exp \left(j K_{N} x\right)}{\left(1-\frac{M K_{N}}{k}\right)} \sin \left[\frac{N \pi}{2 b}(y-b)\right]
$$

For $x>0, p_{\text {ino }}$ can be written as:

$$
\frac{-\rho_{0} \omega}{2 \pi} \int_{-\infty}^{\infty}\left(1-\frac{M K_{N}}{k}\right) \frac{\exp (-j \alpha x)}{\left(\alpha+K_{N}\right)} \cosh \left[\gamma^{\prime}(y-b)\right] d \alpha .
$$

The boundary condition that $v=0$ for $y=b$ for $-\infty<x<0$ yields the result [5] that $A(\alpha)=A_{+}(\alpha), B(\alpha)=B_{+}(\alpha)$, where the subscript + denotes a function free of singularities in an upper halfplane. The Wiener-Hopf technique employs analyticity of functions in appropriately defined upper and lower halfplanes and the notion of analytic continuation. It is appropriate at this stage, therefore, to state precisely what one means by upper and lower halfplanes. As noted earlier, $\gamma$ has branch points at $\pm k$ and $\gamma^{\prime}$ has branch points at $k /(1-M)$ and $-k /(1+M)$. It is well known that in the use of the Wiener-Hopf technique one employs the artifice of assuming initially that $k$ has a small, positive imaginary part and later lets this imaginary part approach zero. With this understanding, an upper halfplane is defined as one for which $\operatorname{Im}(\alpha)<$ $-\operatorname{Im}(k) /(1+M)$ and a lower halfplane is one for which $\operatorname{Im}(\alpha)<\operatorname{Im}(k)$. We restrict the whole analysis to subsonic Mach numbers $(M<1)$; this is more than sufficient to ensure that there is a region of overlap between the upper and lower halfplanes, namely the strip $-\operatorname{Im}(k) /(1+M)<\operatorname{Im}(\alpha)<\operatorname{Im}(k)$.

Continuity of transverse acoustic particle displacement for $x>0$ ensures that $\left[A_{+}(\alpha)-B_{+}(\alpha) /(1+M \alpha / k)\right]$ is analytic in a lower halfplane. But inspection of $\left\{A_{+}(\alpha)-\right.$ $\left.B_{+}(\alpha) /(1+M \alpha / k)\right\}$ shows that it itself is analytic in an upper halfplane. Hence it is an entire function of $\alpha$. By placing appropriate restrictions on the behavior of $\phi$ (cf. [5]) and using Liouville's theorem, we may conclude that the entire function $\left\{A_{+}(\alpha)-\right.$ $\left.B_{+}(\alpha) /(1+M \alpha / k)\right\}$ is, in fact, zero. Continuity of acoustic pressure for $x>0$ gives:

$$
\frac{A_{+}(\alpha)}{\gamma^{\prime}}\left(1+\frac{M \alpha}{k}\right)^{2}\left\{\begin{array}{l}
\operatorname{coth}\left(\gamma^{\prime} b\right) \\
\tanh \left(\gamma^{\prime} b\right)
\end{array}\right\}+\frac{j}{(2 \pi)^{1 / 2}}\left(1-\frac{M K_{N}}{k}\right)\left(\frac{1}{\alpha+K_{N}}\right) \frac{A_{+}(\alpha)}{\gamma}=M_{-}(\alpha),
$$

where $M_{-}(\alpha)$ signifies a function free of singularities in a lower halfplane. In writing down (21) use has been made of the fact that $B_{+}(\alpha)=(1+M \alpha / k) A_{+}(\alpha)$.

Now consider the even and odd cases separately. In the case of $N$ even, we may rewrite (21) as:

$$
\begin{gathered}
\frac{A_{+}(\alpha)\left[1+\frac{M \alpha}{k}\right]^{2} b}{\gamma^{\prime 2} b} \frac{\left[\frac{\gamma^{\prime 2}}{\gamma} \sinh \left(\gamma^{\prime} b\right)+\gamma^{\prime}\left(1+\frac{M \alpha}{k}\right)^{2} \cosh \left(\gamma^{\prime} b\right)\right]}{\sinh \left(\gamma^{\prime} b\right)\left(1+\frac{M \alpha}{k}\right)^{2}} \\
\quad+\frac{j b}{(2 \pi)^{1 / 2}}\left(1-\frac{M K_{N}}{k}\right) \frac{1}{\left(\alpha+K_{N}\right)}=b M_{-}(\alpha) .
\end{gathered}
$$

The next step in attempting to solve this problem by the Wiener-Hopf technique would be to try and factor the term multiplying $A_{+}(\alpha)$ as a product of two terms, one analytic in an upper halfplane and the other analytic in a lower halfplane. As Carrier [2] and Koiter [4] point out, this factorization is often not at all easy and constitutes the most 
serious obstacle to solving problems by Wiener-Hopf techniques. Both Carrier and Koiter point out that often if the exact kernel (we use the word "kernel" to denote the function of $\alpha$ that multiplies $A_{+}(\alpha)$ ) is replaced by another kernel which matches the exact kernel reasonably well, it may be quite easy to factor the approximate kernel. This procedure is adopted in the present paper. Only part of the kernel multiplying $A_{+}(\alpha)$ is so replaced and the replacement is carried out according to guidelines suggested by Carrier [2].

Consider the term $\left\{\left(\gamma^{\prime 2} / \gamma\right) \sinh \left(\gamma^{\prime} b\right)+\gamma^{\prime}(1+M \alpha / k)^{2} \cosh \left(\gamma^{\prime} b\right)\right\} /(1+M \alpha / k)^{2}$ $=P(\alpha)$. Consider its possible replacement by

$$
P^{*}(\alpha)=\frac{\gamma^{\prime} \exp \left(\gamma^{\prime} b\right)(\alpha+2 \delta)}{2(\alpha+\delta)} .
$$

More will be said shortly concerning $\delta$. It is easily verified that $P(0)=P^{*}(0)=-j k$ - $\exp (-j k b)$ and that both $P(\alpha), P^{*}(\alpha)$ tend to $\left[\frac{1}{2} \gamma^{\prime} \exp \left(\gamma^{\prime} b\right)\right]$ as $\alpha \rightarrow \infty$. As suggested by Carrier, we choose $\delta$ so that $d P / d \alpha, d P^{*} / d \alpha$ agree at $\alpha=0$. As a result of a somewhat tedious calculation, the matching of $d P / d \alpha$ and $d P^{*} / d \alpha$ at $\alpha=0$ yields

$$
\delta=(k / 2 M)(1+j \cot (k b)) .
$$

Consider now a modified version of (22) with $P(\alpha)$ replaced by $P^{*}(\alpha)$, namely

$$
\begin{array}{r}
\frac{A_{+}(\lambda)\left(1+\frac{M \alpha}{k}\right)^{2}\left(\gamma^{\prime} b\right) e^{\gamma_{\prime} b}(\alpha+2 \delta)}{[\alpha(1+M)+k] 2(\alpha+\delta) \sinh \left(\gamma^{\prime} b\right)}+\frac{j b}{(2 \pi)^{1 / 2}} \frac{[\alpha(1-M)-k]\left(1-\frac{M K_{N}}{k}\right)}{\left(\alpha+K_{N}\right)} \\
=b[\alpha(1-M)-k] M_{-}(\alpha) .
\end{array}
$$

Let

$$
\frac{2(\alpha+\delta) \sinh \left(\gamma^{\prime} b\right)}{(\alpha+2 \delta)\left(\gamma^{\prime} b\right) \exp \left(\gamma^{\prime} b\right)}=L(\alpha)=L_{+}(\alpha) L_{-}(\alpha)
$$

where $L_{+}(\alpha)$ is a function analytic in an upper halfplane and $L_{-}(\alpha)$ is analytic in a lower half plane. The term $(\alpha+\delta) /(\alpha+2 \delta)$ is part of $L_{+}(\alpha)$ if $\cot (k b) \geq 0$ and is part of $L_{-}(\alpha)$ if $\cot (k b)<0$. Then, by a standard application of the Wiener-Hopf procedure using Liouville's theorem, etc.,

$$
A_{+}(\alpha)=\frac{j b}{(2 \pi)^{1 / 2}} \frac{L_{-}\left(-K_{N}\right)\left[k+K_{N}(1-M)\right]\left(1-\frac{M K_{N}}{k}\right)}{\left(\alpha+K_{N}\right)\left(1+\frac{M \alpha}{k}\right)^{2}} L_{+}(\alpha)[\alpha(1+M)+k] .
$$

Thus $\phi(x, y)$ for $y>b$ is:

$$
\begin{aligned}
& \phi=\frac{-j b L_{-}\left(-K_{N}\right)\left[k+K_{N}(1-M)\right]\left(1-\frac{M K_{N}}{k}\right)}{2 \pi} \\
& \cdot \int_{-\infty}^{\infty} \frac{L_{+}(\alpha) \exp [\gamma(b-y)-j \alpha x][\alpha(1+M)+k] d \alpha}{\gamma\left(\alpha+K_{N}\right)\left(1+\frac{M \alpha}{k}\right)^{2}} .
\end{aligned}
$$


Consider now the case of $N$ odd. In this case

$$
\begin{gathered}
\frac{A_{+}(\alpha)\left(1+\frac{M \alpha}{k}\right)^{2}}{\gamma^{\prime}} \frac{\left[\frac{\gamma^{\prime}}{\gamma} \cosh \left(\gamma^{\prime} b\right)+\left(1+\frac{M \alpha}{k}\right)^{2} \sinh \left(\gamma^{\prime} b\right)\right]}{\left(1+\frac{M \alpha}{k}\right)^{2} \cosh \left(\gamma^{\prime} b\right)} \\
\quad+\frac{j}{(2 \pi)^{1 / 2}}\left(1-\frac{M K_{N}}{k}\right) \frac{1}{\left(\alpha+K_{N}\right)}=N_{-}(\alpha),
\end{gathered}
$$

where $N_{-}(\alpha)$ is a function analytic in a lower halfplane.

In a spirit similar to the even case we replace the term $Q(\alpha)=\left(\gamma^{\prime} / \gamma\right) \cosh \left(\gamma^{\prime} b\right)+$ $(1+M \alpha / k)^{2} \sinh \left(\gamma^{\prime} b\right) /(1+M \alpha / k)^{2}$ by $Q^{*}(\alpha)=\exp \left(\gamma^{\prime} b\right)\left(\alpha+2 \delta^{\prime}\right) / 2\left(\alpha+\delta^{\prime}\right)$, where

$$
\delta^{\prime}=(k / 2 M)[1-j \tan (k b)] .
$$

This ensures that $Q(0)=Q^{*}(0)=\exp (-j k b), d Q / d \alpha=d Q^{*} / d \alpha$ at $\alpha=0$ and that both $Q(\alpha), Q^{*}(\alpha) \rightarrow \frac{1}{2} \exp \left(\gamma^{\prime} b\right)$ as $\alpha \rightarrow \infty$.

Now consider the approximate version of (28), namely:

$$
\begin{aligned}
& \frac{A_{+}(\alpha)\left(1+\frac{M \alpha}{k}\right)^{2} e^{\gamma^{\prime b}}\left(\alpha+2 \delta^{\prime}\right)}{(\alpha(1+M)+k)^{1 / 2} 2\left(\alpha+\delta^{\prime}\right) \cosh \left(\gamma^{\prime} b\right)} \\
& \quad+\frac{j}{(2 \pi)^{1 / 2}} \frac{(\alpha(1-M)-k)^{1 / 2}\left(1-\frac{M K_{N}}{k}\right)}{\left(\alpha+K_{N}\right)}=M_{-}(\alpha)(\alpha(1-M)-k)^{1 / 2} .
\end{aligned}
$$

As before, we factorize $2\left(\alpha+\delta^{\prime}\right) \cosh \left(\gamma^{\prime} b\right) / \exp \left(\gamma^{\prime} b\right)\left(\alpha+2 \delta^{\prime}\right)=K(\alpha)$ as $K_{+}(\alpha) K_{-}(\alpha)$. The term $\left(\alpha+\delta^{\prime}\right) /\left(\alpha+2 \delta^{\prime}\right)$ belongs to $K_{+}(\alpha)$ if $\tan (k b) \leq 0$ and to $K_{-}(\alpha)$ if $\tan (k b)>0$. Application of the Wiener-Hopf method and use of Liouville's theorem now leads to:

$$
A_{+}(\alpha)=\frac{-j K_{+}(\alpha)[\alpha(1+M)+k]^{1 / 2}}{(2 \pi)^{1 / 2}\left(\alpha+K_{N}\right)\left(1+\frac{M \alpha}{k}\right)^{2}}\left(1-\frac{M K_{N}}{k}\right) K_{-}\left(-K_{N}\right)\left[-k-K_{N}(1-M)\right]^{1 / 2} \text {. }
$$

Therefore, in this case $\phi(x, y)$ for $y>b$ is:

$$
\begin{aligned}
& \frac{j}{2 \pi}\left(1-\frac{M K_{N}}{k}\right) K_{-}\left(-K_{N}\right)\left[-k-K_{N}(1-M)\right]^{1 / 2} \\
& \cdot \int_{-\infty}^{\infty} \frac{K_{+}(\alpha)[\alpha(1+M)+k]^{1 / 2} \exp [\gamma(b-y)-j \alpha x] d \alpha}{\gamma\left(\alpha+K_{N}\right)\left(1+\frac{M \alpha}{k}\right)^{2}} .
\end{aligned}
$$

3. Factorization of $L(\alpha)$ and $K(\alpha)$. We recall that

$$
L(\alpha)=\frac{2(\alpha+\delta)}{(\alpha+2 \delta)} \frac{\sinh \left(\gamma^{\prime} b\right)}{\left(\gamma^{\prime} b\right) \exp \left(\gamma^{\prime} b\right)}
$$

where $\delta=(k / 2 M)(1+j \cot (k b))$ and $\gamma^{\prime}=\left(\alpha^{2}-(\alpha M+k)^{\prime 2}\right]^{1 / 2}$. As noted earlier, if $\cot (k b) \geq 0$ then $(\alpha+\delta) /(\alpha+2 \delta)$ belongs to $L_{+}(\alpha)$, otherwise it belongs to $L_{-}(\alpha)$. Now

$$
\gamma^{\prime}=\left(\alpha^{\prime 2}-k^{\prime 2}\right)^{1 / 2},
$$


where

$$
\alpha^{\prime}=\alpha\left(1-M^{2}\right)^{1 / 2}-\left(k M /\left(1-M^{2}\right)^{1 / 2}\right)
$$

and

$$
k^{\prime}=k /\left(1-M^{2}\right)^{1 / 2}
$$

Noble [5] has explained how to factorize a function of type $\sinh (\gamma b) \exp (-\gamma b) /(\gamma b)$ into functions analytic in $\operatorname{Im}(\alpha)>-\operatorname{Im} k$ and $\operatorname{Im}(\alpha)<\operatorname{Im} k$. We may adopt the same formulae to the factorization of $\sinh \left(\gamma^{\prime} b\right) \exp \left(-\gamma^{\prime} b\right) /\left(\gamma^{\prime} b\right)$ except that the plus function will now be analytic for $\operatorname{Im}(\alpha)>\operatorname{Im}(-k / 1+M)$ and the minus function for $\operatorname{Im}(\alpha)<+\operatorname{Im}(k / 1-M)$. This is, of course, entirely satisfactory from the present standpoint. Noble [5] has made the interesting and useful observation that so long as one is interested in $\left|L_{+}(\alpha)\right|$ or $\left|L_{-}(\alpha)\right|$, very simple results may be obtained by the infinite product theorem for meromorphic functions. We merely cite the results from chapter III of Noble's book that, for real $\alpha$,

$$
\begin{aligned}
\left|L_{+}(\alpha)\right| \sim \exp \left[-\frac{b \gamma^{\prime}}{\pi} \cos ^{-1}\left(\alpha^{\prime} / k^{\prime}\right)\right] \exp \left[-/ \alpha^{\prime} b\right]\left|\frac{\sinh \left(\gamma^{\prime} b\right)}{\left(\gamma^{\prime} b\right)}\right|^{1 / 2} \\
\cdot\left|\prod_{r=1}^{N^{\prime}} \frac{\frac{k}{\left(1-M^{2}\right)}\left[1-\left(1-M^{2}\right)\left(\frac{r \pi}{k b}\right)^{2}\right]^{1 / 2}-\frac{k M}{1-M^{2}}+\alpha}{\frac{k}{1-M^{2}}\left[1-\left(1-M^{2}\right)\left(\frac{r \pi}{k b}\right)^{2}\right]^{1 / 2}+\frac{k M}{1-M^{2}}-\alpha}\right|^{1 / 2}
\end{aligned}
$$

where $N^{\prime}$ denotes the upper limit of numbers $r=1,2, \cdots N^{\prime}$ for which $1>\left(1-M^{2}\right)$ $(r \pi / k b)^{2}$. Expression (35) would be multiplied on the right-hand side by $(\alpha+\delta) /(\alpha+2 \delta)$ or not according as $\cot (k b) \geq 0$ or $\cot (k b)<0$. The factorization of

$$
K(\alpha)=2\left(\alpha+\delta^{\prime}\right) /\left(\alpha+2 \delta^{\prime}\right) \exp \left(-\gamma^{\prime} b\right) \cosh \left(\gamma^{\prime} b\right)
$$

where

$$
\delta^{\prime}=(k / 2 M)(1-j \tan k b),
$$

is similarly accomplished and for real $\alpha$, one has:

$$
\begin{aligned}
& \left|K_{+}(\alpha)\right| \sim \exp \left[-\frac{1}{2} \alpha^{\prime} b\right] \exp \left[-\frac{\gamma^{\prime} b}{\pi} \cos ^{-1}\left(\frac{\alpha^{\prime}}{k^{\prime}}\right)\right]\left|\cosh \left(\gamma^{\prime} b\right)\right| \frac{1}{2} \\
& \left|\prod_{r=1}^{N^{\prime \prime}} \frac{\frac{k}{1-M^{2}}\left(1-\left(1-M^{2}\right)\left(r-\frac{1}{2}\right)^{2}\left(\frac{\pi}{k b}\right)^{2}\right)^{1 / 2}-\frac{k M}{1-M^{2}}+\alpha}{1-M^{2}}\left(1-\left(1-M^{2}\right)\left(r-\frac{1}{2}\right)^{2}\left(\frac{\pi}{k b}\right)^{2}\right)^{1 / 2}+\frac{k M}{1-M^{2}}-\alpha\right|
\end{aligned}
$$

where $N^{\prime \prime}$ denotes the upper limit of integers for which $1>\left(1-M^{2}\right)\left(N^{\prime \prime}-\frac{1}{2}\right)^{2}(\pi / k b)^{2}$. Again, Eq. (36) is to be modified on the right-hand side by the factor $\left(\alpha+\delta^{\prime}\right) /\left(\alpha+2 \delta^{\prime}\right)$ according as $\tan (k b) \leq 0$ or $\tan (k b)>0$.

4. Far-field directivity and discussion of results. Examination of the solutions (27) and (32) shows that in the integrand in the integral representation for $\phi$, the only space-dependent term is the term exp $[\gamma(b-y)-j \alpha x]$. If a polar coordinate system is introduced with origin at $y=b, x=0$ then (for $y>b$ ) from Fig. $1,(y-b)=r \sin \theta$ 


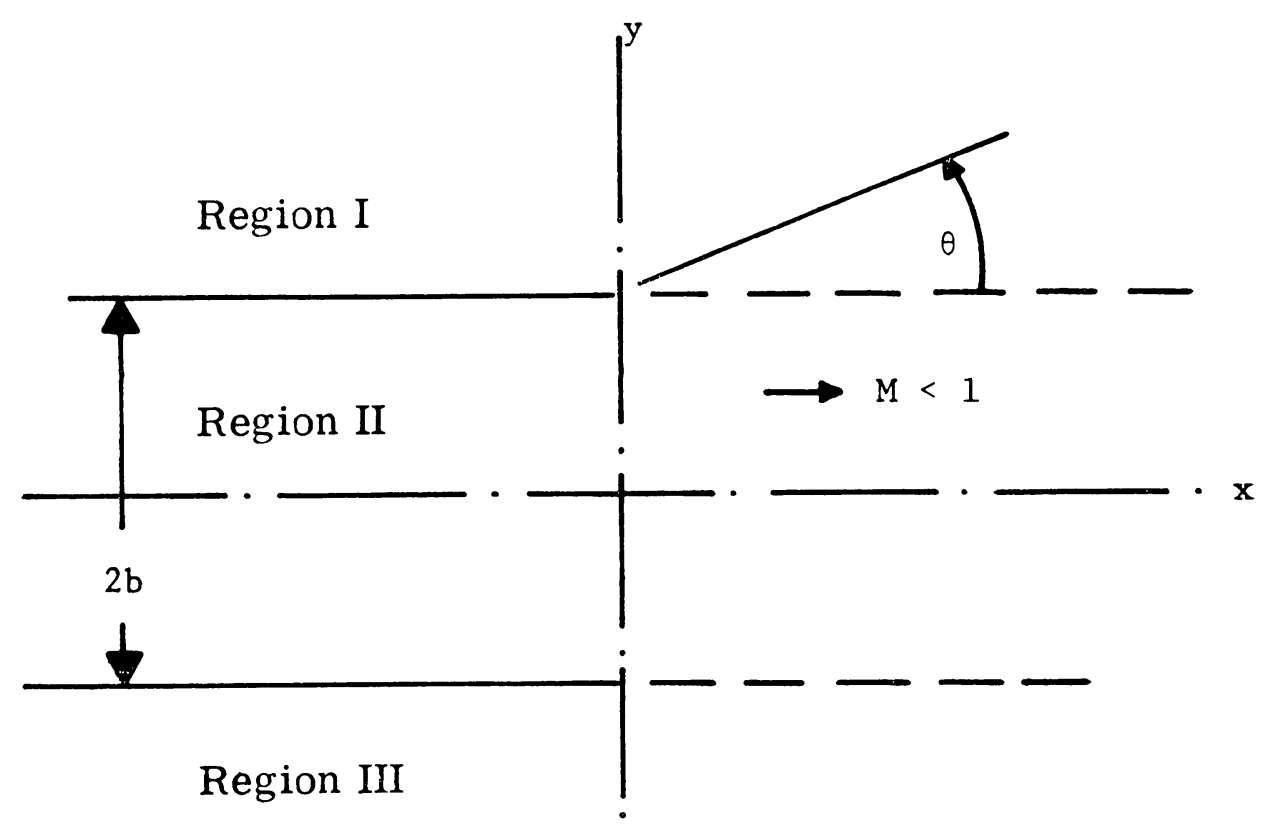

Fig. 1. Definition sketch.

and $x=r \cos \theta$. The term $\exp [\gamma(b-y)-j \alpha x]=\exp [-\gamma r \sin \theta-j \alpha r \cos \theta]$. Thus for large $r$, the dominant contribution to the integral for $\phi(x, y)$ comes from the neighbourhood of the value of $\alpha$ for which $-[\gamma \sin \theta+j \alpha \cos \theta]$ is stationary, which is $\alpha=$ $-k \cos \theta$. This result is fully explained in Noble [5] and Gottlieb [3]. To summarize, the far-field radiation pattern can be obtained from (27), (32) by substituting $\alpha=-k \cos \theta$ in the integrand less the $\exp [\gamma(b-y)-j \alpha x]$ term and then multiplying the result by $\sin \theta$. Both Noble [5] and Gottlieb [3] have shown this and Gottlieb [3] in particular shows how other singularities of $\alpha$ contribute only terms negligible compared to the above term for large $r$. The full expression for the radiation pattern is written out in the Appendices.

There are three nondimensional parameters on which the answer depends, namely $k b, M$ (the exhaust Mach number) and $N$. In Figs. 2-8, plots are presented of such directivity patterns for fixed values of $k b, N$ as a function of $M$ for four values of $M$ of $0,0.3,0.6$ and 0.9 . These plots have all been renormalized so that the peak of the radiation pattern is assigned a value of $100 \mathrm{db}$. Examination of the expressions for the radiation pattern with flow in Appendix 2 reveals at least four discernible flow effects. First, the exponential factor which attenuates the back-scattering, namely

$$
\exp \left[\frac{1}{2} k b \cos \theta\left(1-M^{2}\right)^{1 / 2},\right.
$$

is less effective at the higher Mach numbers. This may be termed a simple refraction effect tending to enhance the back-scattering slightly. Secondly (especially for $M \leq 0.6$ ) the whole pattern is shifted from the axis $(\theta=0)$ to $\theta=\theta_{0}=\cos ^{-1}(1 / 1+M)$. For example, for a nonzero $N$ a node occurs at $\theta_{0}$ instead of at $\theta=0$. In Appendix 3 we have tabulated the nodes of the radiation patterns for the twenty-eight cases studied in 


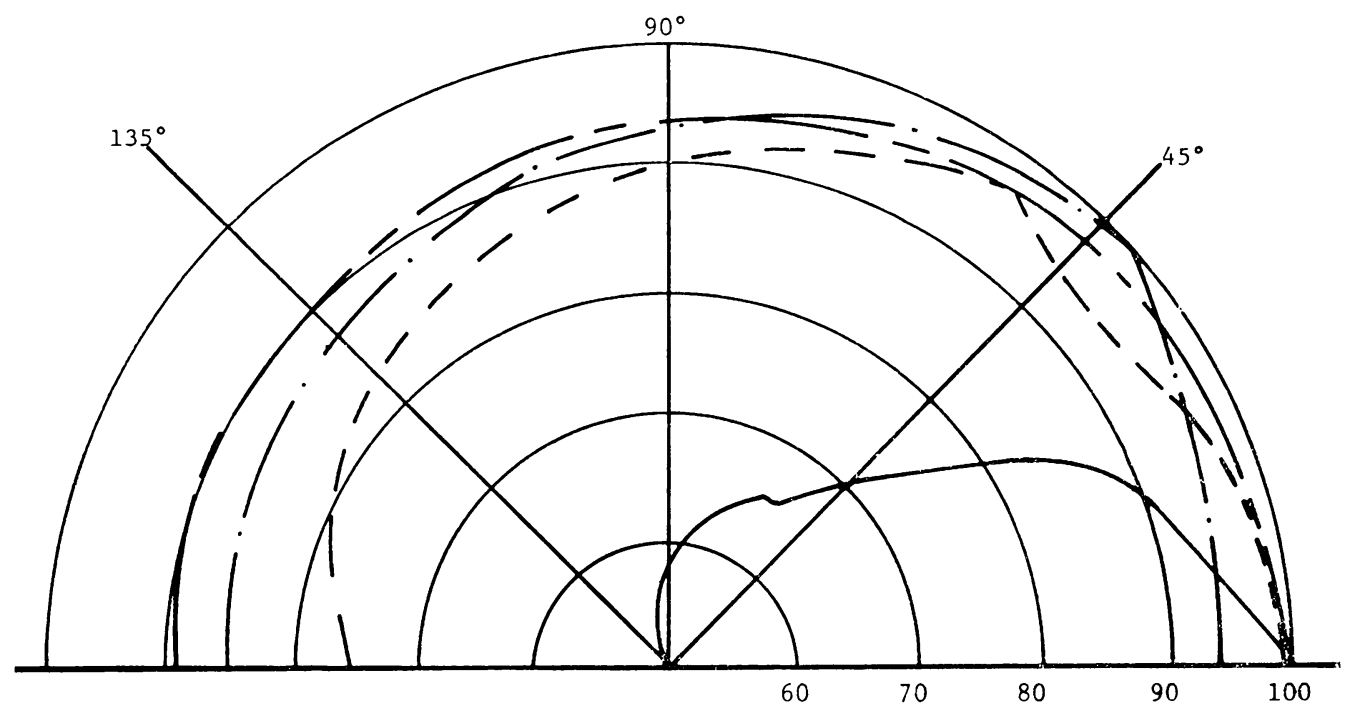

Fig. 2. $\mathrm{kb}=1.25, N=0,-M=0.9,---M=0.6,-\cdots-M=0.3,---M=0$.

Figs. 2-8. Note also the exponentially attenuating factor

$$
\exp \left[-k b\left(\cos ^{2} \theta-(1-M \cos \theta)^{2}\right)^{1 / 2}\right]
$$

in the zone of relative silence $0 \leq \theta \leq \theta_{0}$. Third, the solutions display a substantial "source convection" effect, as indicated by the $1 /(1-M \cos \theta)^{2}$ term, which is frequencyindependent and markedly enhances the forward radiation. This effect may be understood if one notes that the acoustic field in the region $y>b$ and $-\infty<x<\infty$ may be regarded

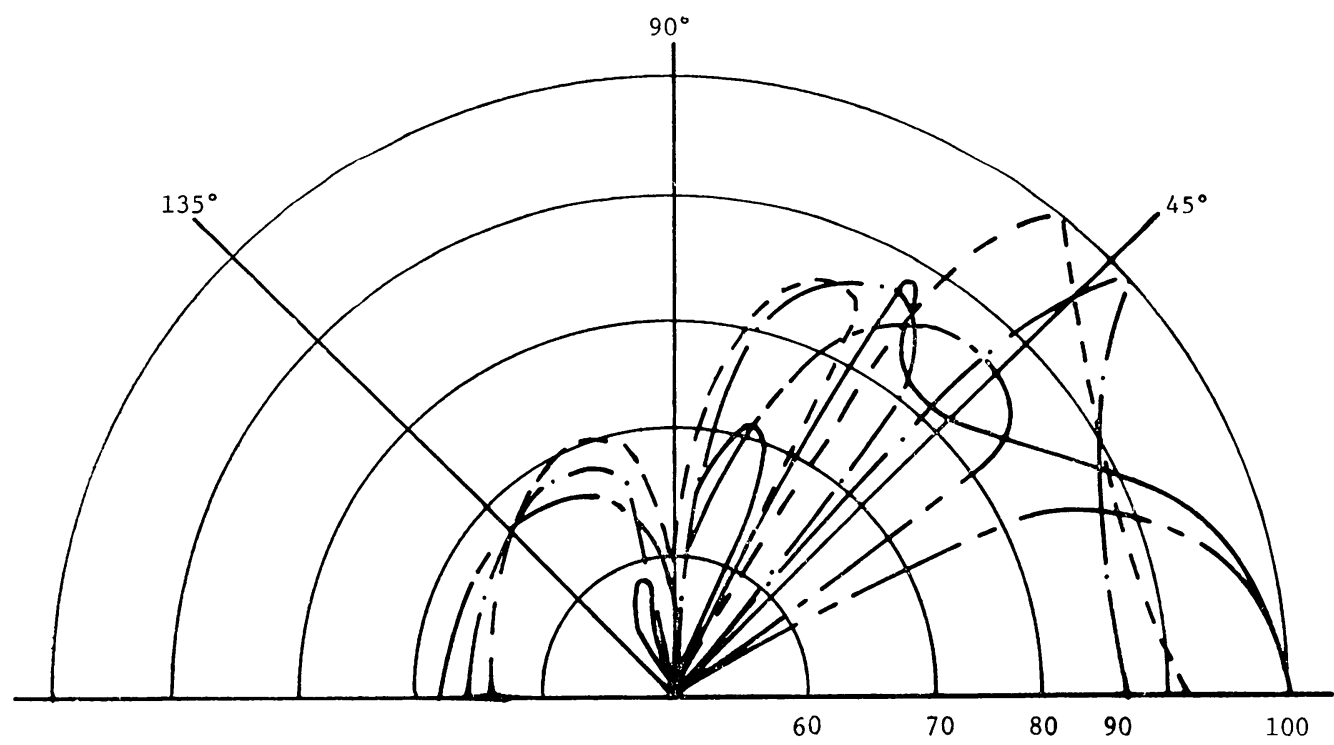

Fig. 3. $\mathrm{kb}=3.75, N=0,-M=0.9,----M=0.6,-\cdots M=0.3,---M=0$. 


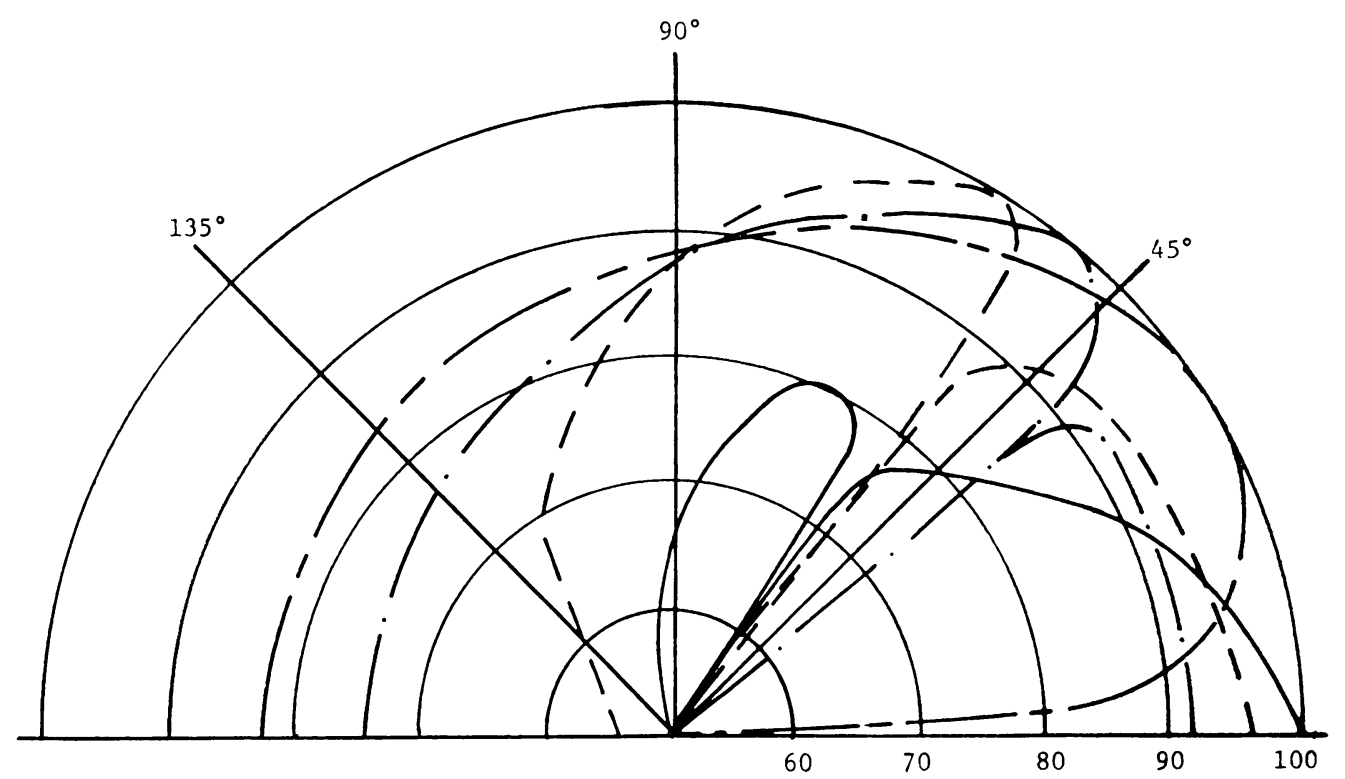

FIG. 4. $\mathrm{kb}=3.75, N=1,-M=0.9,---M=0.6,-\cdots-M=0.3,---M=0$.

as generated by dipole acoustic sources on the plate $y=b$ and $-\infty<x \leq 0$ and by acoustic sources moving along the jet/still-air interface. (To envision the latter, one can see how the incident duct waveguide mode causes a rippling of the jet/still-air interface which then causes a radiated field in the region $y>b$ and $-\infty<x<\infty$.) The interfacial moving sources' directivity will display a source convection effect. This source convection effects dominates the $M=0.9$ radiation pattern even at $k b=6.25$.

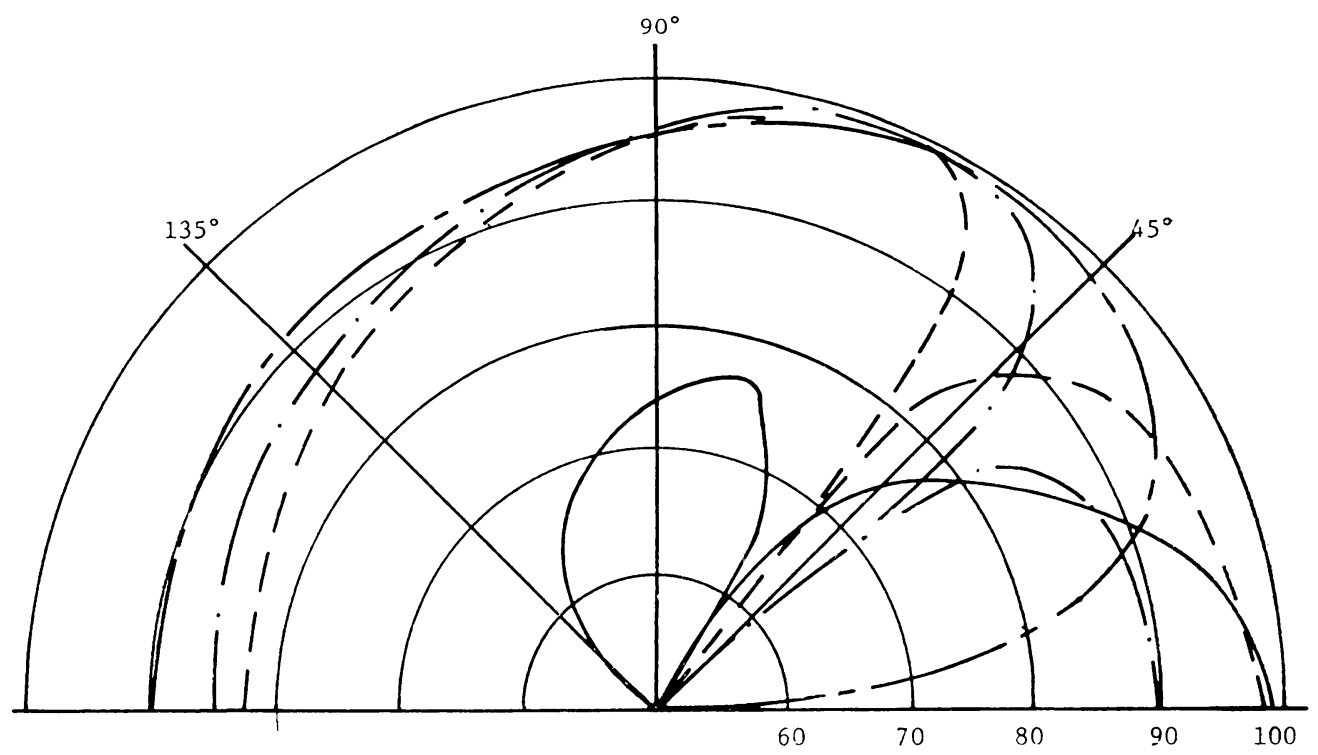

FIG. 5. $\mathrm{kb}=3.75, N=2,-M=0.9,---M=0.6,-\cdots-M=0.3,---M=0$. 


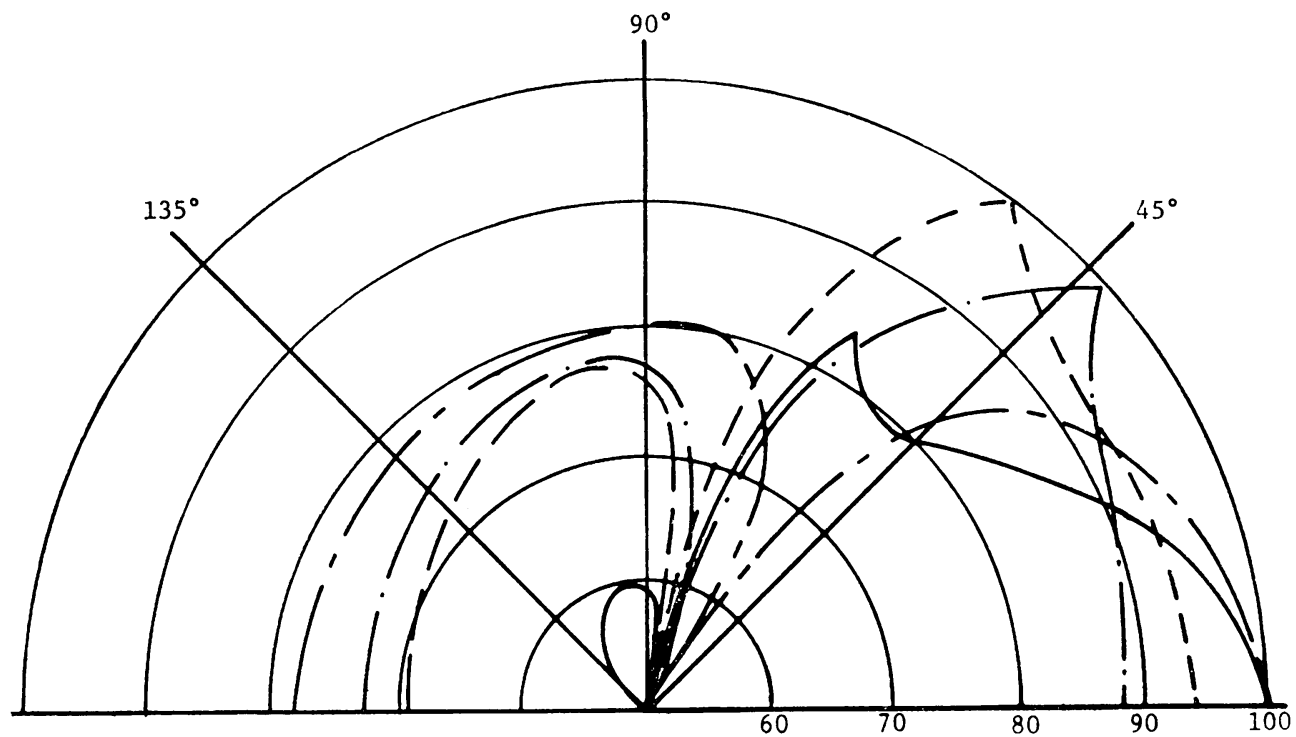

FIG. 6. $\mathrm{kb}=6.25, N=0, \longrightarrow M=.9---M=.6,-\cdot-M=.3---M=0$.

Finally, an effect of the flow (cf. table in Appendix 3) is to increase the number of nodes in the radiation pattern and particularly to crowd together successive nodes.

Some concluding remarks are in order concerning the flow model used and the mathematical methods employed. The steady-flow model used is a "top hat" profile and we may anticipate that such a profile exaggerates refraction effects as compared to realistic, smooth shear profiles. Note, however, the strong refraction effects even at $M=0.3$. Regarding the use of the approximate method due to Carrier and Koiter, one

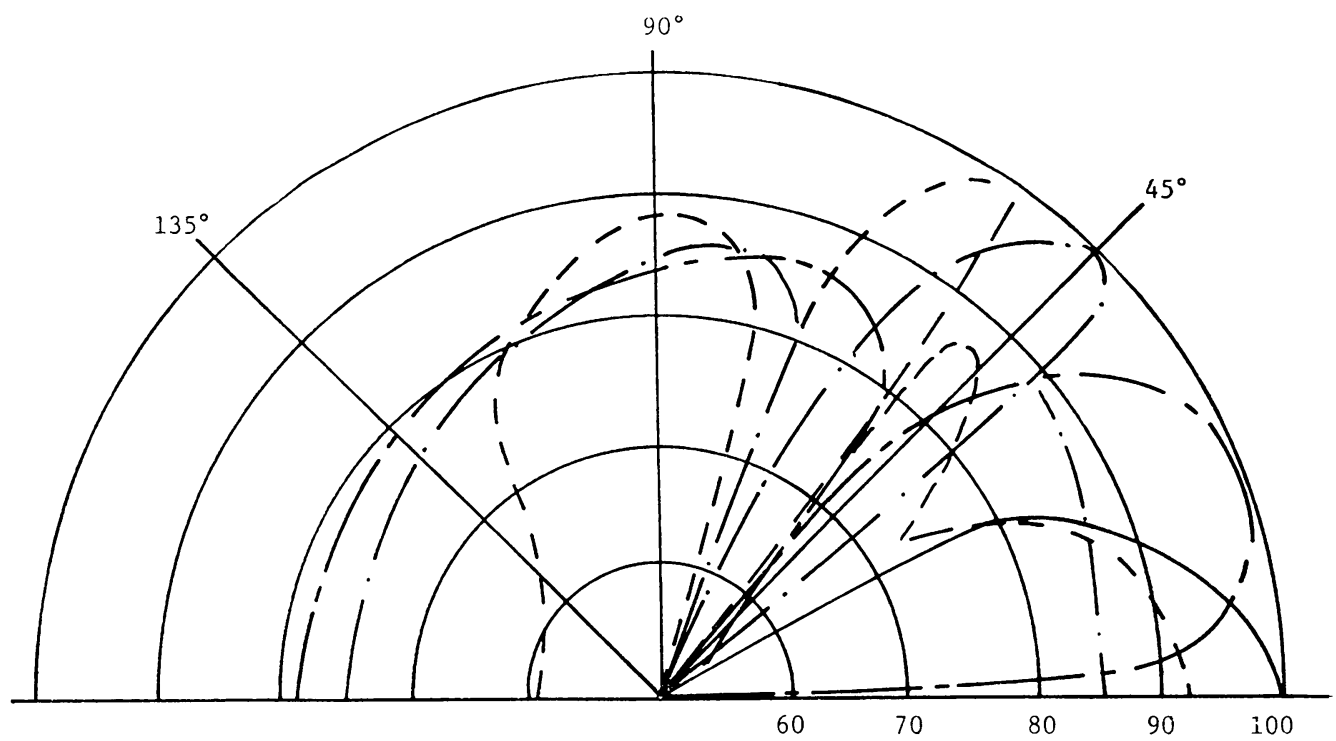

FIG. 7. $\mathrm{kb}=6.25, N=1, \longrightarrow M=.9,---M=.6,-\cdot-M=.3,--\square M=0$. 


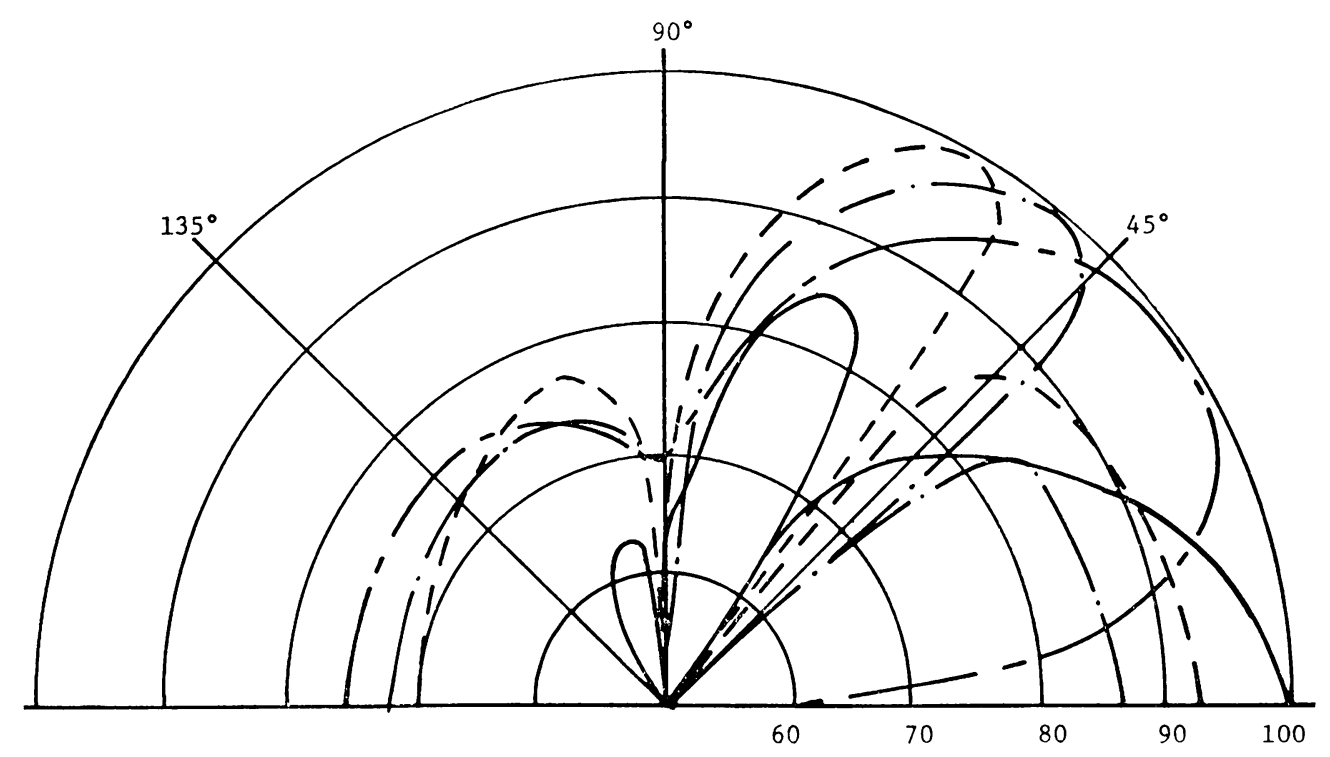

Fig. 8. $\mathrm{kb}=6.25, N=2, \longrightarrow M=.9,--\longrightarrow M=.6,-\cdot-M=3,--\longrightarrow M=0$.

could investigate its numerical accuracy by comparing $Q(\alpha), Q^{*}(\alpha)$ and $P(\alpha), P^{*}(\alpha)$ for $-\infty<\alpha<\infty$ and real $\alpha$, as Koiter has done. We have not done this but the several examples studied by Carrier by this method suggest the approximate procedure is quite accurate. In Appendix 4, a conversion procedure is indicated whereby one can approximately handle a spinning mode problem emitted from an annular duct. Tyler and Sofrin [7] have demonstrated the utility of this procedure for the zero flow problem.

5. Reflection coefficient when $k b<\pi\left(1-M^{2}\right)^{1 / 2}$. If $k b$ is less than $\pi\left(1-M^{2}\right)^{1 / 2}$ it makes sense to consider the reflection coefficient also. In this event, only one duct waveguide mode is propagating. This is the plane wave mode, i.e. $N=0$. In the expression (13) for $p$ in region II upon closing the contour in the upper halfplane (for $x<0$ ) one finds the integrand is free of branch points and has only simple poles at the zeros of $\gamma^{\prime} \sinh \left(\gamma^{\prime} b\right)$. These poles of course correspond to the duct waveguide modes in region II for $x<0$. The lowest upper halfplane pole of $\gamma^{\prime} \sinh \left(\gamma^{\prime} b\right)$ is relevant here when $k b<$ $\pi\left(1-M^{2}\right)^{1 / 2}$ and occurs when $\alpha=k /(1-M)$. The amplitude of the reflected pressure wave is readily calculable and the amplitude of the reflection coefficient for the pressure wave turns out to be:

$$
\begin{array}{ll}
|R|=\exp \left(\frac{-k b}{\left(1-M^{2}\right)^{1 / 2}}\right)\left\{1+\frac{\left(2 M+M^{2}\right) \sin ^{2}(k b)}{1+\left(M^{2}-2 M\right) \cos ^{2}(k b)}\right\}^{1 / 2}, \quad 0 \leq k b \leq \pi / 2, \\
|R|=\exp \left(\frac{-k b}{\left(1-M^{2}\right)^{1 / 2}}\right)\left\{1-\frac{\left(2 M-M^{2}\right) \sin ^{2}(k b)}{1+\left(M^{2}+2 M\right) \cos ^{2}(k b)}\right\}^{1 / 2}, \quad \pi / 2<k b \leq \pi .
\end{array}
$$

The reason for two different expressions in the ranges $0 \leq k b \leq \pi / 2$ and $\pi / 2 \leq k b \leq \pi$ is the use of the Carrier-Koiter method which in the present problem assigns the quantity $\delta$ (Eq. (23)) to the upper halfplane if $\cot (k b) \geq 0$ and to the lower halfplane if $\cot (k b)<0$.

These results are plotted for fixed $k b$ as a function of Mach number in Figs. $9 \mathrm{a}$ and $\mathrm{b}$. The Mach numbers to which the results are plotted are restricted by $\mathrm{kb}<\pi\left(1-M^{2}\right)^{1 / 2}$. 

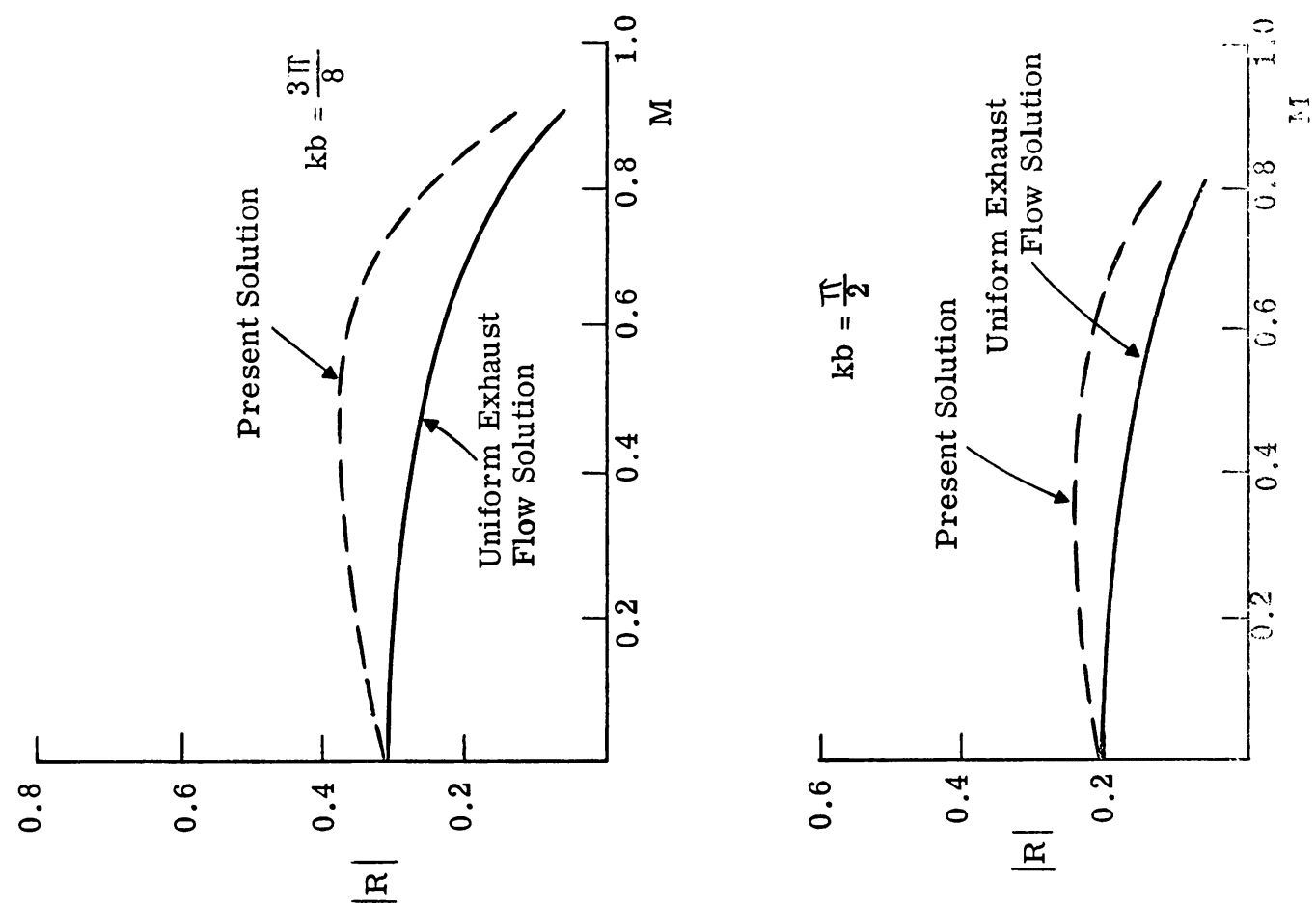

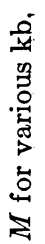
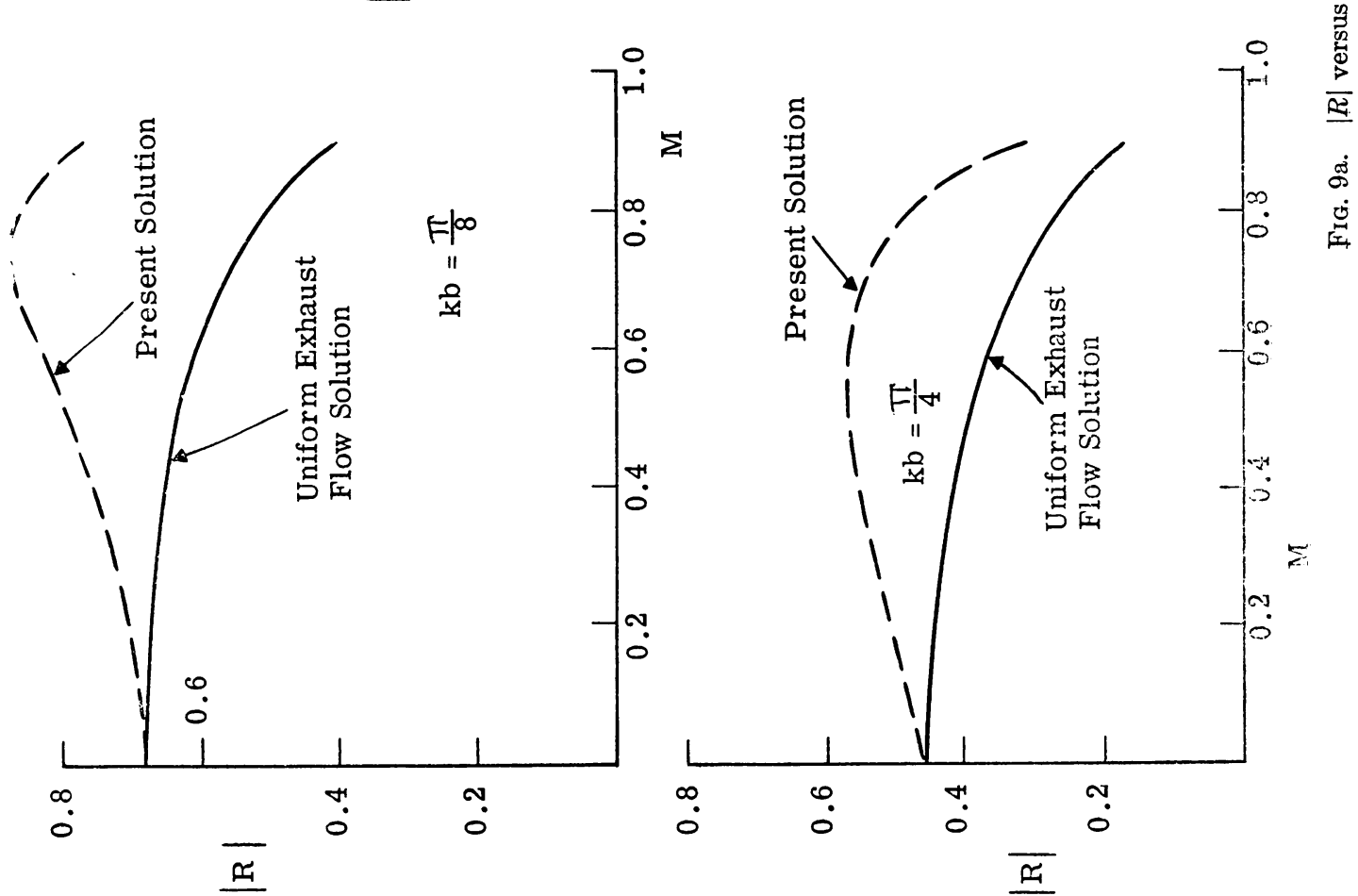


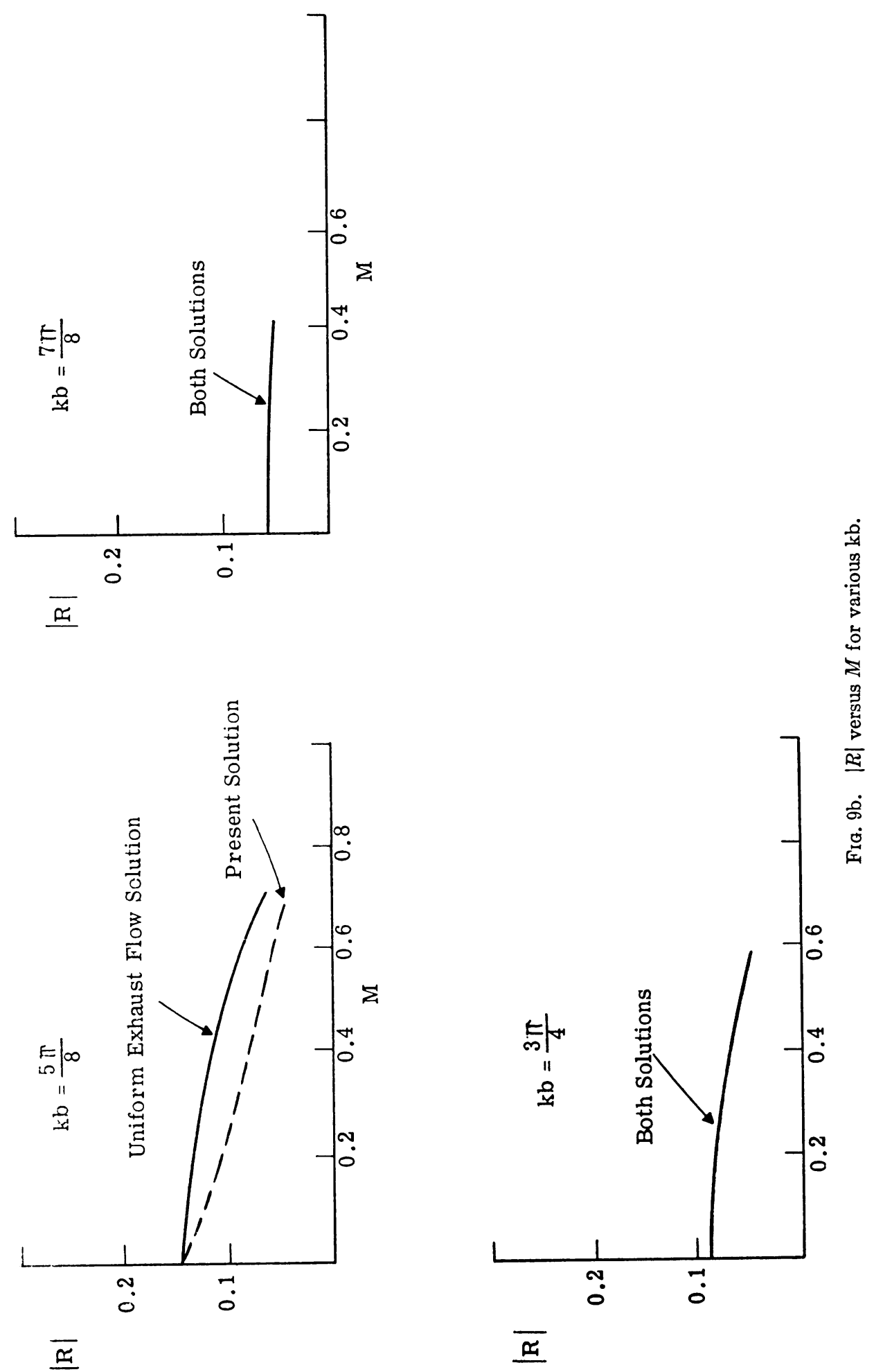




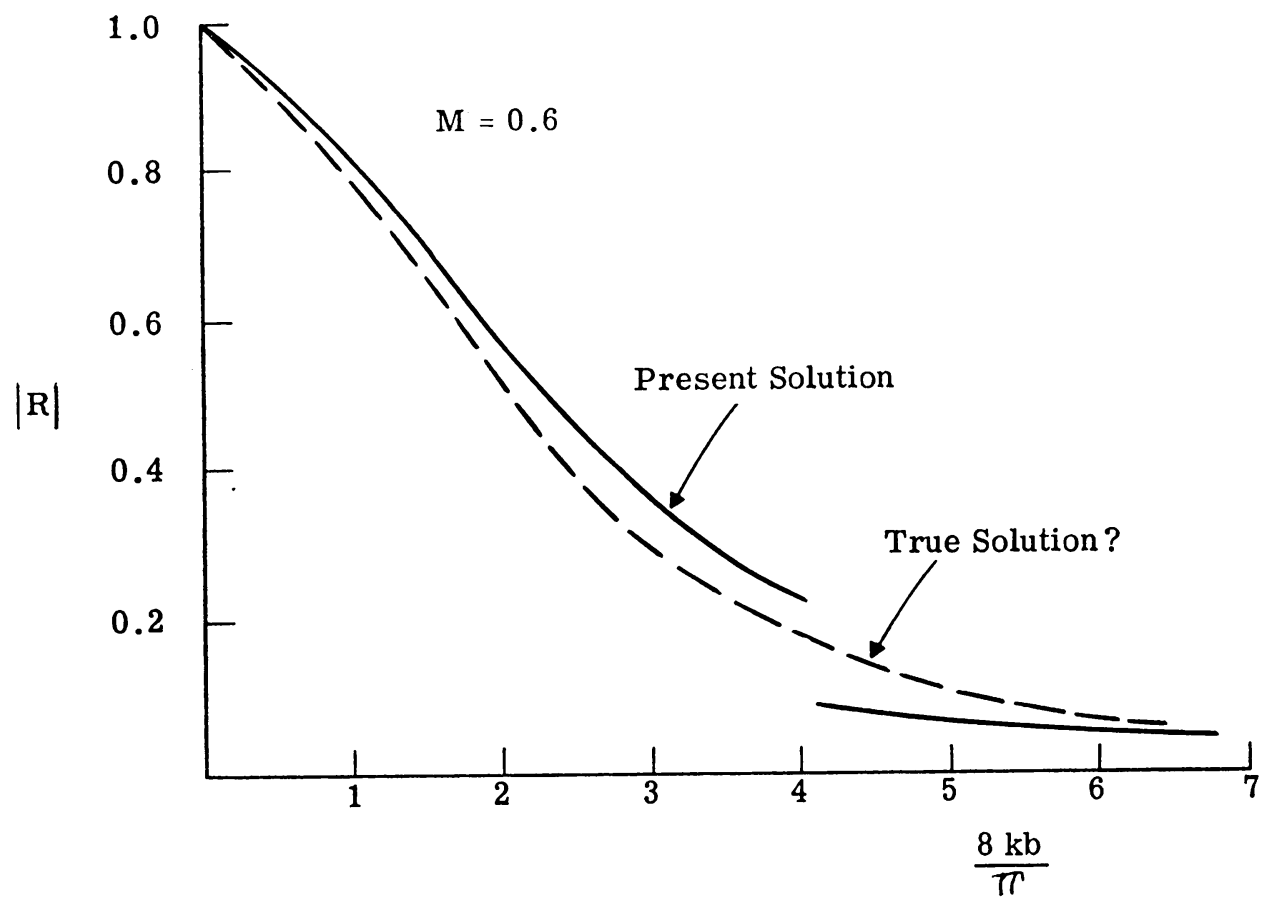

Fig. 10. $|R|$ versus $\mathrm{kb}$ for $M=0.6$.

Also shown on these figures as the "uniform exhaust flow solution" is the solution that one would get if a uniform exhaust flow was assumed over all space, i.e. in regions I and III in addition to II in Fig. 1. The circular tube waveguide version of this problem was considered by Carrier [1] and the result in the present case would be

$$
|R|=\exp \left(-k b /\left(1-M^{2}\right)^{1 / 2}\right) .
$$

As Fig. 9a shows, for $k b \leq \pi / 2$ the problem considered in the present paper has a higher reflection coefficient than the uniform exhaust flow problem. At $k b=5 \pi / 8$ the present problem has a lower reflection coefficient than the uniform flow problem. At $k b=3 \pi / 4$ and $7 \pi / 8$ the two results are virtually indistinguishable and are not shown separately. This change in behavior as $k b$ exceeds $\pi / 2$ is, as noted earlier, a consequence of the use of the approximate method due to Carrier and Koiter. To bring this out in sharper focus we plot $|R|$ against $k b$ for $M=0.6$ in Fig. 10. The curve exhibits a discontinuity at $k b=\pi / 2$. It is not clear whether this is a genuine physical result or is merely induced by the use of the approximate method.

Practically, however, as Figs. 9a and b show, the exhaust jet problem (the problem studied in this paper) shows in general a higher reflection coefficient than either the no-flow or the uniform-exhaust-flow problem. The physical reason appears to be that with an exhaust jet, one has at the exit plane an acoustic mismatch in addition to a geometric mismatch. A uniformly flowing medium with the vector flow Mach number $\mathbf{M}$ may be regarded as an anisotropic acoustic medium in which the velocity of propagation of acoustic plane waves is $c(1+\mathbf{M} \cdot \mathbf{n})$, where $\mathbf{n}$ is a unit vector parallel to the direction of wave propagation. The geometric mismatch is the same whether one considers the 
no-flow or the uniform-flow or the jet-flow problem. Of the three problems, the jet-flow problem alone is characterized by a medium mismatch, and hence it is not surprising that the highest reflection coefficient occurs in general for this case. Regarding the discontinuity in Fig. 10, one should perhaps draw a smooth faired curve through the two segments $0 \leq k b \leq \pi / 2$ and $\pi / 2<k b \leq \pi$. All of this would not in any material sense affect the conclusion that the jet-flow problem in general exhibits a higher reflection coefficient than the uniform-exhaust-flow or the no-flow problem.

Acknowledgements. Partial support of this study under NASA Grant 22-010-063 is gratefully acknowledged. Special thanks are due to Mr. Walter J. Pasko for assistance in programming the computations reported in Figs. 2-8 based on Appendices 1 and 2. The author is grateful to a referee of the original draft of this paper who insisted on a study of the reflection coefficient before final acceptance of the paper. Finally, Drs. S. D. Savkar and M. Kurosaka's comments concerning the significance of the reflection coefficient results were of considerable value.

\section{REFERENCES}

[1] G. F. Carrier, Quart. Appl. Math. 13, 457 (1956)

[2] G. F. Carrier, J. Appl. Phys. 30, 1769-1774 (1959)

[3] P. Gottlieb, J. Acoust. Soc. Amer. 32, 1117 (1960)

[4] W. T. Koiter, Koninkl. Ned. Akad. Wetenschap. Proc. B57, 558 (1954)

[5] B. Noble, Methods based on the Wiener-Hopf technique, Pergamon, 1958

[6] H. S. Ribner, J. Acoust. Soc. Amer. 29, 435 (1957).

[7] J. M. Tyler and T. G. Sofrin, S.A.E. Trans. 70, 309-332 (1962)

Appendix 1: Radiation pattern with no flow. Given $k b, N$ :

Case 1: $\quad N$ even: let $N=2 N^{\prime}$.

1. Let $R$ be an integer such that $R<l k b / \pi<(R+1)$.

2. Let $\theta_{1}, \theta_{2} \cdots \theta_{R}$ be angles defined by $\theta_{r}=\sin ^{-1}(r \pi / k b)$, where $r=1,2, \cdots R$, and $0 \leq \theta_{r} \leq(\pi / 2)$.

3. Let $\phi_{1}, \phi_{2} \cdots \phi_{R}$ be angles such that $\phi_{r}=\left(\pi-\theta_{r}\right)$.

4. For $\theta \neq 0, \theta_{1}, \theta_{2} \cdots \theta_{R}, \phi_{1} \cdots \phi_{R}$ :

$|p(\theta)| \sim \frac{(1-\cos \theta)}{\left.\mid \cos \theta_{N^{\prime}}-\cos \theta\right) \mid} \exp \left(\frac{k b}{2} \cos \theta\right)\left|\frac{\sin (k b \sin \theta)}{k b \sin \theta}\right|^{1 / 2}\left|\prod_{r=1}^{R} \frac{\cos \left(\theta_{r}\right)-\cos \theta}{\cos \theta_{r}+\cos \theta}\right|^{1 / 2}$.

5. If $\theta=0, p=0$ unless $N=0$, in which case it is:

$$
|p(0)| \sim \exp \left(\frac{1}{2} k b\right)\left|\prod_{r=1}^{R} \tan \left(\theta_{r} / 2\right)\right| .
$$

6. If $\theta=\theta_{r}, p=0$ unless $r=N^{\prime}$, in which case it is:

$$
\begin{gathered}
\left|p\left(\theta_{N^{\prime}}\right)\right| \sim \frac{\left(1-\cos \theta_{N^{\prime}}\right)}{\sqrt{ } 2\left|\sin \left(\theta_{N^{\prime}}\right)\right|} \exp \left(\frac{1}{2} k b \cos \theta_{N^{\prime}}\right) \\
\left|\prod_{r=1}^{R} \frac{\cos \left(\theta_{r}\right)-\cos \left(\theta_{N^{\prime}}\right)}{\cos \left(\theta_{r}\right)+\cos \left(\theta_{N^{\prime}}\right)}\right|^{1 / 2} .
\end{gathered}
$$


7. If $\theta=\phi_{m}, p$ is:

$\left|p\left(\phi_{m}\right)\right| \sim \frac{\left(1-\cos \left(\phi_{m}\right)\right)}{\left|\cos \left(\theta_{N^{\prime}}\right)-\cos \left(\phi_{m}\right)\right|}$

$$
\cdot \exp \left[\frac{k b}{2} \cos \left(\phi_{m}\right)\right] \sqrt{ } 2 \cot \left(\phi_{m}\right)\left|\prod_{r=1}^{R} \frac{\cos \left(\theta_{r}\right)-\cos \left(\theta_{m}\right)}{\cos \left(\theta_{r}\right)+\cos \left(\phi_{m}\right)}\right|^{1 / 2} \cdot
$$

Case 2: $\quad N$ odd, let $N^{\prime}=\frac{1}{2}(N+1)$.

1. Let $R$ be an integer such that $\left(R-\frac{1}{2}\right)<k b / \pi<\left(R+\frac{1}{2}\right)$.

2. Let $\theta_{1}, \cdots \theta_{R}$ be angles defined by: $\theta_{r}=\sin ^{-1}\left[\left(r-\frac{1}{2}\right)(\pi / k b)\right]$.

3. Let $\phi_{1}, \phi_{2} \cdots \phi_{R}$ be defined by $\phi_{r}=\left(\pi-\theta_{r}\right)$.

4. For $\theta \neq 0, \theta_{1}, \cdots \theta_{R}, \phi_{1}, \cdots \phi_{R}$;

$|p(\theta)| \sim \frac{\sin (\theta / 2)}{\left|\cos \theta_{N^{\prime}}-\cos \theta\right|} \exp \left(\frac{k b \cos \theta}{2}\right)|\cos (k b \sin \theta)|^{1 / 2}\left|\prod_{r=1}^{R} \frac{\cos \left(\theta_{r}\right)-\cos \theta}{\cos \left(\theta_{r}\right)+\cos \theta}\right|^{1 / 2}$.

5. For $\theta=0, p=0$.

6. For $\theta=\theta_{m}, p=0$ unless $m=N^{\prime}$, in which case it is:

$\left|p\left(\theta_{N^{\prime}}\right)\right| \sim \sin \left(\frac{\theta_{N^{\prime}}}{2}\right) \exp \left[\frac{k b \cos \theta_{N^{\prime}}}{2}\right]\left|\frac{k b}{2 \sin \left(\theta_{N^{\prime}}\right)}\right|^{1 / 2}\left|\prod_{\text {excl. } N^{\prime}}^{R} \frac{\cos \left(\theta_{r}\right)-\cos \left(\theta_{N^{\prime}}\right)}{\cos \left(\theta_{r}\right)+\cos \left(\theta_{N^{\prime}}\right)}\right|^{1 / 2}$.

7. For $\theta=\phi_{m}$ :

$\left|p\left(\phi_{m}\right)\right| \sim \frac{\sin \left(\phi_{m} / 2\right)}{\cos \left(\theta_{N^{\prime}}\right)-\cos \left(\phi_{m}\right)} \exp \left[\frac{k b}{2} \cos \left(\phi_{m}\right)\right]$

$$
\cdot\left|\frac{2 k b \cos ^{2}\left(\phi_{m}\right)}{\sin \left(\phi_{m}\right)}\right|^{1 / 2}\left|\prod_{\substack{r=1 \\ \text { excl. } m}}^{R} \frac{\cos \left(\theta_{r}\right)-\cos \left(\phi_{m}\right)}{\cos \left(\theta_{r}\right)+\cos \left(\phi_{m}\right)}\right|^{1 / 2} .
$$

Appendix 2: Radiation pattern with flow. Given $k b, N, M$, Let $\left.\theta_{0}=\cos ^{-1}(1+M)\right)$.

Case 1: $N$ even, let $N=2 N^{\prime}$.

A: $\cot (k b)<0$.

1. Let $R$ be an integer such that $R<k b / \pi\left(1-M^{2}\right)^{1 / 2}<(R+1)$.

2. Define for $r=1,2 \cdots R$, a sequence of numbers:

$$
R_{r \pm}=\frac{1}{\left(1-M^{2}\right)}\left\{\left[1-\left(1-M^{2}\right)\left(\frac{r \pi}{k b}\right)^{2}\right]^{1 / 2} \mp M\right\} .
$$

3. Let $Q=1 /(1+M)$ if $N=0$ and $R_{N^{\prime}}$ if $N \neq 0$.

4. For all $\left|R_{r \pm}\right|<1$, define angles $\theta_{1}, \theta_{2} \cdots \theta_{R} ; \phi_{1}, \cdots \phi_{R}$ such that $\theta_{r}=\cos ^{-1}\left(R_{r+}\right)$ and $\phi_{r}=\pi-\cos ^{-1}\left(R_{r-}\right)$.

5. For $0 \leq \theta<\theta_{0}$ :

$|p(\theta)| \sim\left[\frac{\cos \theta-\cos }{\cos \theta-Q} \theta_{0}\right]$

$\cdot \exp \left[-k b\left(\cos ^{2} \theta-(1-M \cos \theta)^{2}\right)^{1 / 2}\right]\left|\frac{\sinh \left[k b\left(\cos ^{2} \theta-(1-M \cos \theta)^{2}\right)^{1 / 2}\right]}{k b\left(\cos ^{2} \theta-(1-M \cos \theta)^{2}\right)^{1 / 2}}\right|^{1 / 2}$

$\frac{\exp \left[\frac{1}{2} k b \cos \theta\left(1-M^{2}\right)^{1 / 2}\right]}{(1-M \cos \theta)^{2}}\left|\prod_{r=1}^{R} \frac{R_{r+}-\cos \theta}{R_{r-}+\cos \theta}\right|^{1 / 2}$ 
6. For $\theta=\theta_{0}, p=0$ unless $N^{\prime}=0$, in which case it is:

$$
\left|p\left(\theta_{0}\right)\right| \sim \exp \left\{\frac{1}{2} k b \frac{1-M}{1+M}\right\}(1+M)^{2}\left|\prod_{r=1}^{R} \frac{R_{r+}-\frac{1}{1+M}}{R_{r-}+\frac{1}{1+M}}\right|^{1 / 2}
$$

7. For $\theta_{0}<\theta \leq \pi, p$ in general is:

$$
\begin{gathered}
|p(\theta)| \sim\left|\frac{\cos \theta-\cos \theta_{0}}{\cos \theta-Q}\right| \mid \frac{\sin \left\{k b\left[(1-M \cos \theta)^{2}-\cos ^{2} \theta\right]^{1 / 2}\right\}}{k b\left[\left(1-\left.M \frac{\left.\cos \theta)^{2}-\cos ^{2} \theta\right]^{1 / 2}}{\cos }\right|^{1 / 2}\right.\right.} \\
\frac{. \exp \frac{\left(\frac{1}{2} k b \cos \theta\left(1-M^{2}\right)^{1 / 2}\right)}{(1-M \cos \theta)^{2}}}{\left(\left.\prod_{r=1}^{N} \frac{R_{r+}-\cos \theta}{R_{r-}+\cos \theta}\right|^{1 / 2}\right.}
\end{gathered}
$$

8. If $\theta=\theta_{m}, p=0$ unless $m=N^{\prime}$ : in which case:

$$
\begin{aligned}
&\left|p\left(\theta_{N^{\prime}}\right)\right| \sim \frac{\left(\cos \left(\theta_{N^{\prime}}\right)-\cos \theta_{0}\right)}{\left[1-M \cos \left(\theta_{N^{\prime}}\right)\right]^{2}} \exp \left\{\frac{k b}{2} \cos \theta_{N^{\prime}}\left(1-M^{2}\right)^{1 / 2}\right\} \\
& \cdot\left(\frac{k b}{N^{\prime} \pi}\right)\left|\frac{M+\cos \theta_{N^{\prime}}\left(1-M^{2}\right)}{R_{N^{\prime}+}+R_{N^{\prime}-}}\right|^{1 / 2}\left|\prod_{r=1}^{R} \frac{R_{r+}-\cos \theta_{N^{\prime}}}{R_{r-}+\cos \theta_{N^{\prime}}}\right|^{1 / 2}
\end{aligned}
$$

9. If $\theta=\phi_{m}$, then $p$ is:

$p\left(\phi_{m}\right)\left|\frac{\cos \left(\phi_{m}\right)-\cos \left(\theta_{0}\right)}{\cos \left(\phi_{m}\right)-Q}\right| \frac{\exp \left[\frac{k b}{2} \cos \left(\phi_{m}\right)\left(1-M^{2}\right)^{1 / 2}\right]}{\left[1-M \cos \left(\phi_{m}\right)\right]^{2}}\left(\frac{k b}{m \pi}\right)$

$$
\left|\left[M+\cos \left(\phi_{m}\right)\left(1-M^{2}\right)\right]\left(R_{m+}+R_{m-}\right)\right|^{1 / 2}\left|\prod_{r=1}^{R} \frac{R_{r+}-\cos \left(\phi_{m}\right)}{R_{r-}+\cos \left(\phi_{m}\right)}\right|^{1 / 2}
$$

B: If $\cot (k b) \geq 0$, multiply all previous expressions by the term:

$$
\left|\frac{(1-2 M \cos \theta)^{2}+\cot ^{2}(k b)}{(1-M \cos \theta)^{2}+\cot ^{2}(k b)}\right|^{1 / 2} \text {. }
$$

Case 2: $N$ odd: let $N^{\prime}=(N+1) / 2$ :

A: $\tan (k b)>0$ :

1. Let $R$ be an integer such that $\left(R-\frac{1}{2}\right)<k b / \pi\left(1-M^{2}\right)^{1 / 2}<\left(R+\frac{1}{2}\right)$.

2. Define for $r=1,2, \cdots R$ a sequence of numbers:

$$
R_{r \pm}=\frac{1}{1-M^{2}}\left\{\left[1-\left(1-M^{2}\right)\left(r-\frac{1}{2}\right)^{2}\left(\frac{\pi}{k b}\right)^{2}\right]^{1 / 2} \mp M\right\} .
$$

3. For those $R_{r \pm}$ such that $\left|R_{r \pm}\right|<1$ define angles $\theta_{1}, \cdots \theta_{R}, \phi_{1}, \phi_{2} \cdots \phi_{R}$ such that $\theta_{r}=\cos ^{-1}\left(R_{r+}\right)$ and $\phi_{r}=\pi-\cos ^{-1}\left(R_{r-}\right)$

4. Let $Q=R_{N^{\prime}+}$.

5. For $0 \leq \theta<\theta_{0}$ :

$|p(\theta)| \sim \frac{(\cos \theta(1+M)-1)^{1 / 2} \exp \left[\frac{k b}{2} \cos \theta\left(1-M^{2}\right)^{1 / 2}\right]}{(1-M \cos \theta)^{2}(\cos \theta-Q)}$

$$
\cdot \exp \left[-k b\left(\cos ^{2} \theta-(1-M \cos \theta)^{2}\right)^{1 / 2}\right] \cosh ^{1 / 2}\left[k b\left(\cos ^{2} \theta-(1-M \cos \theta)^{2}\right)^{1 / 2}\right]
$$




$$
\cdot\left|\prod_{r=1}^{R} \frac{R_{r+}-\cos \theta}{R_{r-}+\cos \theta}\right|^{1 / 2} .
$$

6. For $\theta=\theta_{0}, p=0$.

7. For $\theta_{0}<\theta \leq \pi$ : in general, $p$ is

$|p(\theta)| \sim \frac{(1-\cos \theta(1+M))^{1 / 2} \exp \left[\frac{k b}{2} \cos \theta\left(1-M^{2}\right)^{1 / 2}\right]}{(1-M \cos \theta)^{2}(\cos \theta-Q)}$

$$
\cdot\left|\cos \left[k b\left((1-M \cos \theta)^{2}-\cos ^{2} \theta\right)^{1 / 2}\right]\right|^{1 / 2}\left|\prod_{r=1}^{R} \frac{R_{r+}-\cos \theta}{R_{r-}+\cos \theta}\right|^{1 / 2}
$$

8. If $\theta=\theta_{m}, p=0$ unless $m=N^{\prime}$, in which case it is:

$$
\begin{aligned}
\left|p\left(\theta_{N^{\prime}}\right)\right| \sim \frac{\left(1-(1+M) \cos \left(\theta_{N^{\prime}}\right)\right)^{1 / 2} \exp \left[\frac{k b}{2} \cos \left(\theta_{N^{\prime}}\right)\left(1-M^{2}\right)^{1 / 2}\right]}{\left[1-M \cos \left(\theta_{N^{\prime}}\right)\right]^{2}} \\
\cdot\left|\frac{(k b)^{2}\left[M+\left(1-M^{2}\right) \cos \left(\theta_{N^{\prime}}\right)\right]}{\left(N^{\prime}-\frac{1}{2}\right) \pi\left(R_{N^{\prime}+}+R_{N^{\prime}-}\right)}\right|^{1 / 2}\left|\prod_{r=1}^{R} \frac{R_{r+}-\cos \left(\theta_{N^{\prime}}\right)}{R_{r-}+\cos \left(\theta_{N^{\prime}}\right)}\right|^{1 / 2} .
\end{aligned}
$$

9. If $\theta=\phi_{m}, p$ is:

$$
\begin{aligned}
\left|p\left(\phi_{m}\right)\right| & \sim \frac{\left(1-(1+M) \cos \left(\phi_{m}\right)\right)^{1 / 2}}{\left[1-M \cos \left(\phi_{m}\right)\right]^{2}\left(\cos \phi_{m}-Q\right)} \exp \left[\frac{k b}{2} \cos \left(\phi_{m}\right)\left(1-M^{2}\right)^{1 / 2}\right] \\
& \left|\frac{(k b)^{2}\left[M+\cos \left(\phi_{m}\right)\left(1-M^{2}\right)\right]\left(R_{m+}+R_{m-}\right)}{\left(m-\frac{1}{2}\right) \pi}\right|^{1 / 2}\left|\prod_{\substack{r=1 \\
\text { exci. } m}}^{R} \frac{R_{r+}-\cos \left(\phi_{m}\right)}{R_{r-}+\cos \left(\phi_{m}\right)}\right|^{1 / 2} .
\end{aligned}
$$

\begin{tabular}{|c|c|c|c|c|c|c|}
\hline$k b$ & $N$ & $M$ & $\begin{array}{l}\text { No. of } \\
\text { Nodes }\end{array}$ & 1st Node & 2nd Node & 3rd Node \\
\hline \multirow{4}{*}{1.25} & 0 & 0 & 0 & & & \\
\hline & & 0.3 & 0 & & & \\
\hline & & 0.6 & 0 & & & \\
\hline & & 0.9 & 0 & & & \\
\hline \multirow[t]{8}{*}{3.75} & 0 & 0 & 1 & 56.9 & & \\
\hline & & 0.3 & 1 & 70.68 & & \\
\hline & & 0.6 & 1 & 77.16 & & \\
\hline & & 0.9 & 1 & 80.63 & & \\
\hline & 1 & 0 & 1 & 0 & & \\
\hline & & 0.3 & 1 & 39.72 & & \\
\hline & & 0.6 & 1 & 51.32 & & \\
\hline & & 0.9 & 2 & 58.24 & 109.5 & \\
\hline
\end{tabular}

B: If $\tan (k b) \leq 0$, multiply all previous expressions for $p$ by:

$$
\left|\frac{(1-2 M \cos \theta)^{2}+\tan ^{2}(k b)}{(1-M \cos \theta)^{2}+\tan ^{2}(k b)}\right|^{1 / 2} \text {. }
$$

Appendix 3: Nodes $(p=0)$ of radiation patterns (all angles in degrees). 


\begin{tabular}{|c|c|c|c|c|c|c|}
\hline$k b$ & $N$ & $M$ & $\begin{array}{l}\text { No. of } \\
\text { Nodes }\end{array}$ & 1st Node & 2nd Node & 3rd Node \\
\hline \multirow[t]{4}{*}{3.75} & 2 & 0 & 1 & 0 & & \\
\hline & & 0.3 & 1 & 39.72 & & \\
\hline & & 0.6 & 1 & 51.32 & & \\
\hline & & 0.9 & 1 & 58.24 & & \\
\hline \multirow[t]{12}{*}{6.25} & 0 & 0 & 1 & 30.17 & & \\
\hline & & 0.3 & 2 & 50.61 & 91.05 & \\
\hline & & 0.6 & 2 & 60.46 & 90.51 & \\
\hline & & 0.9 & 3 & 66.52 & 90.34 & 140.4 \\
\hline & 1 & 0 & 2 & 0 & 48.94 & \\
\hline & & 0.3 & 2 & 39.72 & 64.29 & \\
\hline & & 0.6 & 2 & 51.32 & 72.02 & \\
\hline & & 0.9 & 3 & 58.24 & 76.47 & 109.5 \\
\hline & 2 & 0 & 1 & 0 & & \\
\hline & & 0.3 & 2 & 39.72 & 91.05 & \\
\hline & & 0.6 & 2 & 51.32 & 90.51 & \\
\hline & & 0.9 & 3 & 58.24 & 90.34 & 140.4 \\
\hline
\end{tabular}

Appendix 4: Approximate analysis of spinning modes. In a spinning mode problem in an annular duct the given inputs will be $k^{\prime} a$ where $k^{\prime}=\omega / c, a=$ mean radius of annulus, $\sigma$ the hub tip ratio, $m$ the tangential lobe number and $N^{\prime}$ the radial mode order. Then the two-dimensional problem which simulates the three-dimensional problem approximately will have:

$$
k b=\frac{k^{\prime} a(1-\sigma)}{(1+\sigma)}\left(1-\frac{m^{2}}{\left(k^{\prime} a\right)^{2}}\right)^{1 / 2}
$$

and an $N$ equal to the number of nodal circles in the mode pressure distribution for the axisymmetric problem. 